Revue scientifique francophone en Communication

organisationnelle

$18 \mid 2000$

Non-verbal, communication, organisation

\title{
Apprentissage de la voix chantée et éducation vocale
}

\section{François Mellet}

\section{OpenEdition}

\section{Journals}

Édition électronique

URL : https://journals.openedition.org/communicationorganisation/2444

DOI : 10.4000/communicationorganisation.2444

ISSN : 1775-3546

\section{Éditeur}

Presses universitaires de Bordeaux

\section{Édition imprimée}

Date de publication : 1 novembre 2000

ISSN : 1168-5549

Référence électronique

François Mellet, "Apprentissage de la voix chantée et éducation vocale ", Communication et organisation [En ligne], 18 | 2000, mis en ligne le 27 mars 2012, consulté le 08 décembre 2022. URL : http://journals.openedition.org/communicationorganisation/2444; DOI : https://doi.org/10.4000/ communicationorganisation. 2444

Ce document a été généré automatiquement le 5 août 2021.

Tous droits réservés 


\title{
Apprentissage de la voix chantée et éducation vocale
}

\author{
François Mellet
}

Communiquer sur le non-verbal signifie la « réflexion » de l'oreille qui croit entendre à la bouche qui est supposée pouvoir, forme évidente d'utopie effleurant ses prémisses imaginées ou tombée dans le réel dont les "mathèmes » apprennent le bricolage d'un monde espéré ailleurs, instantané. Les généalogies des messagers de savoirs arbitrés sont humaines : qu'elles sont les instances d'où il est parlé, écrit, acte ? Comment dire l'incommunicabilité qui en distille le plaisir ? L'être soi ensemble n'est pas plus social que l'être soi seul. Libres d'en appréhender les signes d'aliénation, une névrose nous essaye au verbe de chair en place d'action car les corps sont lourds et attachent les esprits : non assujettis, une lueur d'artifices.

\section{De l'écriture du corps à la génétique vocale}

2 Communication en souffrance des règles de sa génétique, contrats de socialités d'avant l'intelligence machinique des corps sans chair, charge l'auteur d'une illusoire institution; à lui de s'inscrire au sens de ce qui s'échappe de son espace postural, écrit, verbal. Communiquer l'écrit pour l'oral est prétexte d'un verbe spatial. Censurés les bruits, cris et chants, une rhétorique préexiste au scriptural. Toujours points de vue, les lieux, places, figurations réelles ou imaginaires des récipiendaires sont dans l'intention, et les médiums décorporés radiophoniques, télévisuels et télé-informatiques, paramétrés, s'y déploient: le médium est le message, sans les confondre pour en distinguer les effets des corps actants, les causes des intentions, les métaphores des " réalités ", les sensations des métabolismes. Le gestuel pur implique intégralement le coup de dé du tout ou rien au paradigme des langages du corps et de la grande iconicité, à entendre comme hypersensoriel, tactile, sonore, graphique, textuel et visuel. Chanteurs, pédagogues, éducateurs, professionnels de la voix et du geste y gagneraient en santé et longévité. La pédagogie est l'esprit des corps, détente structurée à se tenir, mouvoir et émouvoir, à l'image de Decroux, Freinet, Deligny. 
Lecoq, Oury en théâtre, pédagogie, éducation, psychothérapie. Dire en se taisant oblige, sans tourner en bouche la langue du bégaiement infini, à imploser dans la télépathie ou réfléchir socialement l'émotion. Pour le chanteur le schéma corporel vocal bonifié est pallesthésique et kinesthésique : l'expression spectaculaire exige une maîtrise souple et fine. La voix chantée, corporellement invisible, paraît au béotien plus simple. Le danger de cette perception est ignoré des élèves ou négligé par les formateurs. L'instrument du chanteur est le corps entier, la vibration générale. Pour l'instrumentiste, la logique du corps ne se réduit pas à de trompeuses apparences : la respiration du pianiste apporte au son, les tropes et la linguistique fourbissent le défaut de sa cuirasse à l'espèce parlante pour se mentir à l'autre : magie du spectacle, corriger le lapsus le redouble et mène à son terme le désir qu'il travestit, véritable cauchemar, rêve enfin réussi couronné d'émotions applaudies. Se taire, par défaut d'être entendu, est infantile, parler pour ne rien dire est la réciproque académie des normopathes. Dire de l'extérieur du corps, sans lien organique avec l'architecture intérieure, fait gagner leurs vies aux illusionnistes dans un geste calculé. Le verbe se tient du surcroît de " pouvoir dire ce qu'il ne parle pas », autant que « ce qui ne se dit pas » est en puissance de verbe. Mon intervention exclut de facto une démonstration que je suis ici dans l'impuissance de produire. Un hypertexte in vivo serait plus conforme à mes interventions initiales autour de l'hyperception et des langages du corps, ergonomiques livre-opéras.

3 Je paie ici mon écot aux registres physiques et métaphysiques. Ma pédagogie est de la surprise et chante en de rares moments, grâce accueillie comme un don. Chanter à parole pleine - souffle, timbre, mordant, vibrato, harmoniques, formants - est plus ardu que parler technique. Les signaux codés et «syntaxes" chiffrées des langues animalières, enfantines, professionnelles, secrètes et argotiques sont faciles. Mais les langues des signes sont sonores : le corps est vibration d'air, bruissement de feuilles, battement d'ailes, musique d'oiseaux. La voix et le chant s'inscrivent dans cette filiation phylogénique en une conduite humaine par révolution langagière et anamnèse sensitive : une histoire construit le chanteur, en deçà de son cortex, anticipant les observations de Freud sur la mémoire. L'animalité est enfance de l'art, le professeur métaphysicien des corps, le péripapéticien démonstrateur du mouvement, le Chanteur centaure, Orphée lyre, Thésée Minotaure, Ulysse Personne, les porteurs d'homériques exploits aspirateurs mythologiques en navigation hypertextuelle libre d'avoir été polie de métrique hellène pour un démesure poïétique. Au musée imaginaire du trafiquant d'air, les artistes chantent avec Goethe et Chevreul, experts en couleurs humaines, inspirateurs du beau pour une éducation textuelle, linguistique, corporelle, visuelle, olfactive, posturale. architecturale, écologique, bagage de tout apprenti en compagnonnage en ressources de sensibilité, patrimoine culturel, familial, professionnel. Le maître est autodidacte de son abord du bien parler, chanter, vivre et précepteur habile ouvert aux pluriels pour l'éveil d'excellence vers tous. L'intention et la technique vibrent ensemble.

\section{Mythologies : Comment devient-on maître de parole ?}

4 L'exposé est impertinent sans références métaphoriques, contextuelles ou empruntées ni langue d'invariants. Une fondation appelle le travail de lisibilité d'un statut, mais le danger rôde dans les cabinets et laboratoires des savants exhibant du métalangage désincarné à des clients prostitutionnels. Maître de chant, qu'est ce ? Ecrire un manuel 
est dissolution du corps, l'objet n'est pas un espace réglé sujet aux expériences pour en extraire des généralités. L'enseignement du chant, hyperespace indéchiffrable et fugace ponctué de trouvailles, est une culture ancienne de maitres de savoir-chanter de bouche à oreilles. Le résultat inextricable est révélateur de sociologies plus que d'une physique de la qualité que jauge le cerveau en neuroscience expérimentale. Les contextes épistémologiques poussent désormais d'autres formes qu'inventent de nouvelles générations de formateurs. Sans formation pédagogique socialement lisible, ma démarche, manière cubiste et fractale, s'autorise d'elle-même avec les encouragements entendus de condisciples actifs de maîtres communs. Le paysage s'ouvre pour offrir à des musiciens, non chanteurs solistes, la possibilité d'un certificat d'aptitude pour postuler une place de professeur en un Conservatoire. Mon premier maître de chant fut seulement grande, disent de vrais amateurs musiciens et chanteurs sûrs en goût. J'ai l'audace de croire qu'elle m'a fait le don de deux ou trois petites choses, dont j'ai conservé la trace, développée par l'oreille de son assistant de toujours, compagnon d'excellence. Ce foncier prospère à mon insu de pêcheur d'étoiles avec de récents maîtres, mes élèves de qui j'ai tout appris du reste, anges gardiens de mon entrée dans ce monde, longue et joyeuse marche de l'expérience, soutien de ma névrose d'origine et pratique ambulatoire. Le mot d'une élève, qui parle peu et chantera peutêtre, sur ce qu'elle appelle mon professionnalisme chemine avec la gratuité. Toujours tisser l'âme, mise en abîme du futur antérieur : gratification.

\section{Le non-verbal et la bouche d'or, utopie de l'utopie}

Communiquer une technique par une incorporation de qualité met à la question les intentions de l'auteur de tout ouvrage, transcrites orphiquement. Auteur, l'émetteur est da capo et au creux d'une communication corporelle, gestuelle, sonore, musicale, verbale, événement singulier de réception, chacun reçoit la scène de sa pathie. 11 peut y avoir du meurtre dans le collectif, le théâtre n'est pas la vie. l'enseignement une autre responsabilité. Une performance désigne sa valeur d'intention, proche de l'inutile, hors des savoirs distribués, partagés, incorpores, échanges: scientificité douteuse et nécessaire par choix métisse et l'académisme nous guette, plus grand commun diviseur. Rien n'est acquis d'avance au plaisir de la transmission. La logique est celle d'une communauté à géométrie sociale, transcendée dans l'écart de la marge, entre l'asile fou de la solitude et l'imagerie collective des médias, dessaisissement redevable aux petites variations d'immobilité mouvante. Le projet d'une parole pleine se soutient d'une pulsion de mort, jardin gardien de la vie : Alceste. qui aime les hommes, est au supplice de l'illusion d'être compris, huit mode d'expression est homogène, en rhétorique et style, à l'objet: ici en cours, hors de tous les textes à écrire, il implémente une inquiétante étrangeté. L'émetteur tient du récepteur comme le levier du poids, le pédagogue de l'élève, l'analyste de l'analysant, le sujet de son altérité, le soliloque du colloque. Chaque cerveau est culturellement programmé, nouveau roman sans début ni lin. évapore dans la cartographie implicite de liens mémoriaux.

6 M'adressant récemment a des collègues du secondaire, eux aussi à la question de la transmission de savoirs disciplinaires, sur le thème "Prospectives de l'Utopie: une exigence entre droit et métaphysique». transforme en un exercice de style sur «Utopies et Mémoire». je laissai, passeur de passeurs, les participants à ma provocation. Je suis car l'éthique me travaille aussi, ma façon de ne pas répondre aux 
questions une méthode. Je découvris aussi ma vision de l'utopie et réalisai ma dernière provisoire folie sur l'argument de la Ville-Monde. Gilles Deleuze. familier de la schizophrénie voyageuse et résistante, grand déterritorialiste avec Felix (iuattari. le dit bien dans son hommage à François Chatelet :

« ne pas nous laisser clouer à un centre établi... acquérir la capacité de transporter un centre avec soi pour organiser des ensembles de relations symétriques et réversibles effectuées par des hommes libres... ne pas savoir à I avance comment quelqu'un. éventuellement, se trouvera capable d'instaurer en lui et hors de lui un processus de rationalisation... s'il y a une chance, de quoi quelqu'un a-t-il besoin, comment procède-t-il pour sortir de ses démolitions... nous ne gâcherons aucune chance ».

7 Je m'en amuse encore : sans traces cinématiques de ma pédagogie des signes ni témoins de mes pratiques, l'essentiel de ma pédagogie de la voix chantée est gestuel polymorphe. Je m'engage néanmoins. " pire orgueil que la modestie, ne pas accepter d'être le meilleur ", à rendre audible une longue pratique de laboratoire. Mes élèves sont acteurs d'un laboratoire des «langages du Corps» avec architectes, musiciens, danseurs, acteurs pour un travail chimérique sur les «logiques de la sensibilité et politiques publiques ».

\section{L'anticipation du futur antérieur et le retour aux sources}

Un retour s'impose sur mon fonds lyrique, imaginaire familial par cousinage en continuité d'une formation en psychologie clinique acquise essentiellement au dernier Château de Lavoisier. Ce grand savant déclara « rien ne se perd, rien ne se crée, tout se transforme ». beau principe pour le pédagogue vocal. Il commit aussi un superbe «il n'y a pas de pierres qui tombent du ciel car il n'y a pas de pierres dans le ciel ». Et pourtant elle tourne, dît Galilée pour sauver sa tête. Lavoisier. ancêtre de Bercy, pas si fou. perdit la sienne. On n'a pas fait mieux dans la pédagogie du chant, la recherche suppose des erreurs, des compromissions et des assassinats. Les hôpitaux psychiatriques ne sont pas les meilleurs lieux pour vivre avec les «malades». non normopathes ordinaires et les châteaux ont été lieux de vie, comme un corps pour la voix. Les aléas analytiques m'y confirmaient ce que l'enfant avait appris de son traumatisme d'avoir été mis au monde pour en assumer les conséquences vitales. Chaque soignant se soignait, un peu dépouillé de ses statuts, à la généralité ménagère de la maison avec sa grammatologie particulière, où la déconstruction n'était pas gratuite. J'ai travaillé en ateliers, l'espérance nous tient lieu de nécessité, d'êtres en souffrance d'eux-mêmes, scandant une sémiotique d'espace ouvert, temps tissé par une grille mobile, celle du parc ouvrant sur la cafetière proximale qui en savait toujours plus que les préposés « psychanalystes ». Ne souhaitant pas échoir maître arpenteur du lieu, je fuis ensuite vers une autre chronicité universitaire. J'avais frôlé la petite compagnie de Jacques Lacan à Saint-Anne avant ses Écrits, pas commis pour être lus. la pulsion de mort travaillant le scribe. Le tissage, la cuisine et le dessin transitaient du sens ; la question de la voix m'est devenue une évidence. Les pathologies de ruptures de dires, aphonies, dysphonies. m'inscrivirent didactiquement dans un conservatoire avec l'idée saugrenue d'y acquérir des connaissances techniques: m'exercer en ville au tissage de la voix du corps. Une appétence m'y poussa, anticipant un centre expérimental universitaire de théâtre musical et d'art lyrique avec ses laboratoire et 
studio-école, soufflés par un hommage à mes professeurs. Une démarche neurologique ne peut s'écrire que romancée : la main écrit le cerveau, les cultures les mythologies familiales et phylogéniques sont multiples, polymorphes et incorporées.

\section{Deux ou trois choses sur la pédagogie vocale}

9 L'outillage d'une clinique de la sensibilité socialisée de rôles chantés se distribue en ressources variées : anatomie posturo-respiratoire, laryngologie. phonéto-acoustique, diction déclamatoire, langues, répertoires et styles, pédagogie des passages. L'échange est primordial, triangulé pour l'apprentissage, sans relation directe avec le savoir scientifique ni la dimension métaphorique de l'arsenal pontifié. Il est recommandé qu'il soit humain, in fine parfois chez les professionnels, amateurs en recherche venant d'autres disciplines ou étrangères au dispositif vocal: musiciens, thérapeutes, ingénieurs. Le travail est un méthodique corps à corps d'appropriation de sa voix vive par l'élève, assisté par des sensations internes neurologiques, pallesthésiques et kinesthésiques pour une figuration sonore. L'image posturale accessible par des jeux de miroirs visualise une image mentale en réflexe. Le schéma corporel de la mobilité se développe in vivo pour des performances ordinaires. La recherche d'une qualité spectaculaire au delà du débrouillage demande un perfectionnement du schéma. L'éducation est rééducation, liée à la volonté et la persévérance où les périodes dites critiques, plus variables qu'on ne croit, jouent leurs rôles. La performance vocale est virtualité du corps et l'éducation «maîtrise de la liberté » entre perception, idéation et émotion, équilibre d'antagonismes obligés. Le professeur peut faire chanter les élèves tous de la même façon, avec des résultats moyens, pour ne pas dire plus: il aura tendance à les dire peu intelligents ou pas «chanteurs». ce qui arrive: de bonnes qualités sont préférables ainsi qu'une bonne santé générale, seul le travail m'intéresse. Les professeurs du secondaire savent que leur voix fatigue à certaines périodes : ils sont des "professionnels» chronicisés de la voix et la gestuelle est un élément de la performance pédagogique. Les pratiques corporelles sont sine qua non en danse, chant, acrobatie, cirque, les fautes souvent irréversibles dans l'engagement de zones archaïques de l'être. Une prise de conscience, liée au "vouloir pouvoir devoir », est d'importance pour entendre le travail de l'apprenti chanteur ou en didactique. Le dispositif est un jeu à quatre, hyperespace symétrique d'actions/réactions réversibles en empathies. Des configurations dynamiques peuvent être analysées, chaque situation demeurant singulière. Un chantier de recherches et formations s'ouvre, peu organisé sur le plan scientifique, alors que l'on se plaît à proclamer un supposé «secret de la voix ».

\section{Logiques politique et sensible en formation au spectacle vocal}

Les coordinations se précisent en un travail d'infimes variations énergétiques. Une confiance mal placée conduit à des ruines partielles incompatibles avec des carrières longues. Cet effritement déstabilise, alors que les circuits mal empruntés en gardent les ornières. La patience et la volonté d'un professeur, avec un élève doué de qualités qui méritent le détour, sont les conditions d'apparents miracles, toujours possibles. Toute réussite est une exception et tient à des rencontres fortuites, évidences après coup, sauf 
pour ceux qui savent le travail. L'humilité, l'excellence, la générosité le sous-tendent. Ces professeurs existent, souvent dans l'ombre où se conçoit la lumière, peu nombreux, faillibles aussi: ils sont humains, projections de nos faiblesses d'idéaux, sans autre sanction que l'émotion. Ils se méritent et ne sont pas la majorité, de nombreuses voix disparaissent sans raisons affichées : ce procès anticipe des conséquences néfastes en cascades. Des artistes en fin de carrière s'offrent l'illusoire substitut de la scène avec des élèves. Des chanteurs, usés par une technique moyenne et des carrières courtes mais respectables ont comme recours, ou - le pire n'est jamais sûr comme authentique accomplissement, l'enseignement du chant avec des positionnements narcissiques aléatoires. Jouer sur les coordinations cérébrales suppose de pratiquer sans anesthésie ni souffrance, seulement réponse neuro-musculaire et tolérance psychologique. La pédagogie de la voix chantée est non-verbale sauf accompagnements ordinaires, indications théoriques, métaphoriques et qualitatives comme trames obligées d'un paysage mental incorporé par captures posturales. auditives, palatales, pour une architecture vocale adaptée.

11 La relation de corps à corps est centrale et les apprentissages se jouent pour les acteurs du corps. La technique n'est pas une fin en soi : des interprètes animés des rôles et des auteurs pourront sauver de la désespérance d'un monde réglé. La leçon est collective, le professeur multiple. La finesse d'un narcissisme réussi est nécessaire chez le pédagogue, pris un temps dans les compromis sociaux de son idéal: il peut ne pas pouvoir le meilleur pour ses élèves, sous couvert de dévouement. Il est difficile d'imaginer une formation fiable des professeurs, recrutés le plus souvent à leurs prix. Le système français s'y prête mal: les disciplines artistiques, scientifiques et les humanités ne sont pas présentes dans les mêmes lieux, avec des tutelles différentes. Ce mal pourrait tirer avantage d'un examen de la situation nord-américaine, qui suggère quelques réflexions profitables aux politiques. Des tentatives pour promouvoir la qualité sont apparues, sous des influences extérieures frictionnant les idées reçues et les pouvoirs établis, avec les dangers de l'institué et de la rente. L'étranger est souvent fascinant, jamais parfait, parfois surfait : aucun professeur n'est parfait, il y a de la place pour le travail et la recherche. Les premiers temps de formation sont critiques, comme les premiers instants de la vie, où se joue le destin de la personne. Les analyses laissent place à la discussion, à la polémique et à la fructueuse dispute. Controversées, elle conduiront à repenser l'avenir institutionnel et pédagogique des arts du spectacle vocal et lyrique. Une nouvelle approche corporelle du spectacle vivant est à réfléchir, en intelligence avec les moyens de reproduction et les technologies numériques. La logique d'image, capitalisée au détriment des qualités individuelles et du temps nécessaire à la construction de l'excellence, fait de l'artiste une figure ambiguë, fascinante et critique. Le retour de troupes, jouant une authentique qualité, à laquelle une presse libre et éduquée pourrait contribuer, avec des assises de masse, ménagerait l'hypothèse d'un travail élitaire pour tous, à la Vitez. Engagé dans une pratique ensemblière des qualités de toutes langues, le pari du beau chant, français en particulier avec sa phonétique et une dynamique riche de déséquilibres vibratoires, avec un pragmatisme expérimental et une culture nourrie d'apports étrangers, tisse une filiation de philosophes de la sensibilité. Je laisserai la parole, provisoirement, à Victor Maurel. créateur du Iago de Verdi et de son Falstaff, concluant sa communication à Milan en 1890 :

«Voilà donc le triste état où se trouve l'enseignement actuel du Chant et ainsi

s'explique la crise de laquelle souffre l'art lyrique contemporain. Quel remède 
apporter à ce mal qui vient de ce que l'enseignement est basé tout entier sur l'expérience? Pourquoi ne pas chercher le remède dans la science? Pourquoi ne pas sortir du domaine des effets pour pénétrer dans la sphères des causes ? C'est la conclusion pratique qui se dégage de toute cette conférence : allons à la science !».

Le travail vibratoire du corps, résonnant en économie d'énergie, est long. Les maîtres sont peu nombreux, les infrastructures inadaptées aux ressources. La recherche, la formation et les performances ont vocation à s'y développer en viviers. Une politique publique est possible pour un humanisme des corps revenu des technologies du virtuel. Ni discipline des états ni illusion du laisser faire, la formation est pratique de l'infime et $\mathrm{du}$ clair-obscur, obligeant à de nouvelles copies: la vie est un non-lieu. Pas de pédagogie vocale de qualité hors du travail. Physique et Métaphysique articulent une logique de la sensibilité dont les politiques se doivent le souci. Pour être soi-même ensemble, une assise instable fait avancer les (dés)équilibres : l'endoxalite guette aussi les normopathes du spectacle. Osons nous autoriser de nous-mêmes, entendre ce que nous sommes, pluriel de nos limites, chimérique rhizome généralisé, couture de l'hybride, bricolage de mondes imagés rapiécés de pulsions de mort : l'éthique est la consigne en toute humilité, sans savoir établi.

\section{BIBLIOGRAPHIE}

BERNARD M.. L expressivité du corps, Delarge, 1976.

DELEUZE G., Périclès et Verdi. Editions de Minuit, Paris, 1996.

HUSSON R., La voix chantée. Gauthier-Villars. Paris, 1960.

LE HUCHE F., La voix, Masson. Paris. 1991.

LEIPP E., Acoustique et Musique, Masson. Paris, 1971.

MELLET F., «Pédagogie de la voix chantée et hypermédia », in Le Son et la Voix, sous la direction de Marie Thonon, Médiation et Information nº 8, L'Harmattan, Paris. 1998.

MILLER R., La structure du chant. IPMC. Paris. 1990.

ORMEZZANO Y., Le guide de la voix, Odile Jacob. Paris, 2000.

RONDELEUX L-J., Trouver sa Voix. Le Seuil. Paris. 1974.

SCOTTO DI C, « La voix chantée ». in La Recherche n² 235, Paris, 1991.

VASSE D., L Ombilic et la Voix, Le Seuil. Paris, 1974.

VENNARD W., Singing : the mechanism and the technic. Carl Fisher, New York. 1967.

\section{AUTEUR}

FRANÇOIS MELLET

Université de Paris 8 